\title{
Stirling Engine Nodal Analysis Program
}

\author{
A. Schock* \\ Fairchild Space and Electronics Company, Germantown, Md.
}

\begin{abstract}
A general-purpose program for the nodal analysis of Stirling-eycle devices or other cyclic, regenerative machines is described. It employs finite-difference, explicit-forward solutions and is readily adaptable to various machine topologies. The machine is divided into specifiable numbers of fluid and solid nodes. The program solves three coupled gasdynamic differential equations (mass, momentum, and energy balances) for each fluid node and an energy balance for each solid node. Interactions between these are represented by heat-transfer and fluid friction terms. The kinetic energy of the fluid and the inertial resistance to flow acceleration are accounted for. Leakages through seals and ports are included in the analysis. The equations are solved by means of u modified leapfrog technique, using special schemes for enhanced mathematical stability and for accelerated convergence to a steady-state cycle. The program is applicable to both disciplined-piston and free-piston engines. A typical problem is solved, and the solutions are presented in Eulerian and L.agrangian form.
\end{abstract}

\section{Introduction}

$\mathbf{S}^{\mathrm{N}}$ NAP (Stirling Nodal Analysis Program) is a digital computer program designed to provide detailed analyses of Stirling-cycle devices, such as Stirling engines or refrigerators. The program was developed by Fairchild Industries under a contract from the Department of Energy's Division of Adyanced Systems and Materials Production, in conjunction with a parallel free-piston Stirling engine development contract to Mechanical Technology; Inc.'

Like earlier programs (c.g., Ref. 2), the SNAP program employs finite-difference, explicit-forward solutions and is written in Fortran to permit easy modification and adaptation to various machine topologies. The machine's fluid passages are represented by a one-dimensional nodal string, consisting (in sequence) of a single expansion-space node, specifiable numbers of heater nodes, regenerator nodes, and cooler nodes, and a single compression-space node. In addition to these, there are corresponding parallel strings of metal wall nodes and regenerator matrix nodes. Interactions between adjacent fluid nodes and metal nodes are represented by appropriate heat-transfer and fluid friction terms.

A typical example of such an array, with a total of 25 working-space nodes, is illustrated schematically in Fig. 1. Nodes 26 and 27 represent gas springs required for resonant piston and displacer motions in the free-piston engine to which the program was applied.

For each gas node, the program solves the full set of fluiddynamic equations, consisting of mass, momentum, and energy balances. The energy balance includes a kinetic energy term, and the momentum balance includes a term accounting for inertial resistance to fluid acceleration. No other program incorporating those terms was available in the public literature at the inception of this study. This is no longer so, since the publication of an excellent Ph.D. thesis by Urieli. ${ }^{3}$

An alternative program in which inertial forces are ignored was also developed and will be discussed later in this paper. That program, in effect, assumes instantaneous quasi-equilibrium between the local pressure gradient and the friction drop due to fluid flow. It provides equally detailed but less accurate results than the full inertial program, but its operating speed is one to two orders of magnitude higher. The loss in accuracy depends on machine dimensions, operating

Received Jine 12, 1978; revision received Sepi. 22, 1978. Copyright (9) 1978 by A. Schock. Published by the American Institute of Aeronarties and Astronautics, Inc., with permission.

Index categories: Reciprocating Machinery; Computational Methods; Analytical and Numerical Methods.

'Manager, Energy Systems Department. frequency, and other design parameters, and its magnitude can only be assessed by ultimate comparison of results from both programs for the same design. Although the analysis discussed in this paper deals with an engine with prescribed piston and displacer motions, the program has also been applied to a problem with free-piston dynamics, in which the displacer and piston motions are purely the result of forces exerted by the gas and the mechanical load.

\section{Analytical Model}

The analytical model used is Eulerian (node boundaries fixcd in space) rather than Lagrangian (moving node boundaries enclosing fixed gas masses). However, once the Eulerian solution is obtained, the results can easily be displayed in Lagrangian form. Both iypes of output graphs will be presented: cyclic variations of fixed space points, and cyclic variations of specific gas particles.

The nodes in the finite-difference model are numbered in ascending order from the expansion space $(i=1)$ to the compression space $(i=n)$. Consider a typical gas node $i$ of length $L_{j}$, cross-sectional area $A_{i}$, wall surface area $S_{m i}$, matrix surface $S_{m i,}$, and hydraulic diameter $D$, given by

$$
D_{i}=\frac{4 A_{i}}{\left(S_{i e f}+S_{i w i}\right) / L_{i}}
$$

Node $i$ has a wall temperature $T_{w w}$, matrix temperature $T_{i m i}$, and contains gas at a mean pressure $P_{i}$, density $\rho_{i,}$ and temperature $T_{1}$. For a perfect gas with gas constant $R$, these are related by

$$
P_{S}=p_{i} R T_{i}
$$

The gas in node $f$ has a mean velocity $u_{i}$, with a corresponding volumetric flow rate

$$
V_{i}=A_{i} u_{i}
$$

and mass flow rate

$$
F_{1}=V_{i} p_{i}
$$

Note that $u_{i}, V_{\text {r }}$ and $F_{i}$ are defined as positive when the local flow is in the direction from the expansion space toward the compression space. In addition to the node-center variables. just defined, let us define $\bar{A}_{i}, \bar{P}_{i}, \bar{p}_{i}, \bar{T}_{i}, \bar{u}_{i}, \bar{V}_{i}$, and $\bar{F}_{i}$ as the

tWhere there are multiple ducts in parallel, their tombined crosssectional and surface areas must be used. 


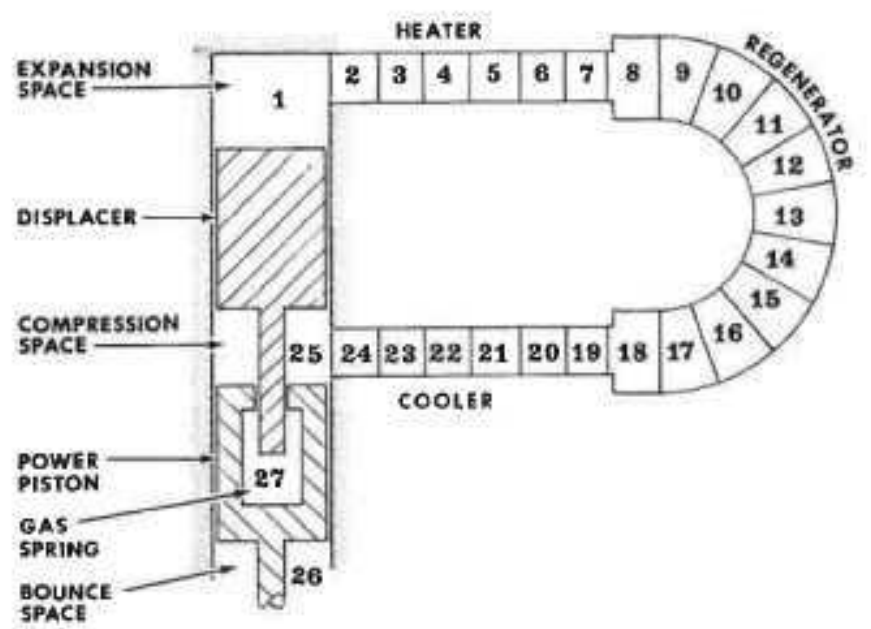

Fig. 1 Nodal model of engine.

corresponding variables at the boundary between node $i-1$ and node $i$. How these are computed in the program will be discussed later.

The gasdynamic differential equations in the program solve for the time variation of the mass $m_{i}$, momentum $M_{i}$, and energy $E_{j}$ of the gas in each node $i$. These three variables are defined by

$$
\begin{gathered}
m_{j}=A_{i} L_{i} \varrho_{j} \\
M_{i}=m_{i} u_{i} \\
E_{i}=m_{i}\left(C_{v} T_{i}+1 / 2 L_{j}^{2}\right)
\end{gathered}
$$

where $C_{v}$ is the constant-volume specific heat of the gas at temperature $T_{i}$.

\section{Finite-Difference Equations for Mass and Momentum}

The rate of change of mass in mode $t$ is equal to the net mass flow rate into the node, so that

$$
\frac{\mathrm{d} m_{i}}{\mathrm{~d} t}=\vec{F}_{i}-\vec{F}_{i+s}
$$

The rate of change of momentum in a typical node is equal to the net momentum flow rate across its two boundaries, plus the net pressure force on the node (in the axial direction), minus the retarding force due to shear at the wall and matrix surfaces. Thus,

$$
\begin{aligned}
& \frac{\mathrm{d} M_{i}}{\mathrm{~d} t}=\left(\hat{F}_{i} \hat{u}_{i}-\hat{F}_{i+i} \hat{u}_{i+i}\right)+\left(\hat{A}_{i} \hat{P}_{i}-\hat{A}_{i+i}, \hat{P}_{i+i}\right) \\
& -\left(\bar{A}_{i}-\hat{A}_{i+i}\right) P_{r}-A_{i}\left(f_{i} L_{i} / D_{i}\right)\left(D_{2} \rho_{i}\left(u_{i} \mid u_{i}\right)\right.
\end{aligned}
$$

where $f_{7}$ is the D'Arcy friction factor. Note that Eq. (9) uses the product $\left|u_{i}\right| u_{i}$ instead of $u_{i}^{2}$, since the friction force direction is always opposite to the flow direction.

One of the shortcomings of the present program is that; even though the fluid flow in the Stirling machine is reciprocating, the computations of the friction factor $f$, and the heat-transfer coefficient $h_{i}$ for each node and each time step are carried out by means of steady-state correlations. However, it is not expected that errors introduced by use of steady-state correlations have very much effect on the overall results. Sensitivity studies carried out with the SNAP program indicate that even factor-of-2 changes in the correlations for friction factor and heat-transfer coefficient had only minor effect on the predicted power output and efficiency.
Friction factors and heat-transfer coefficients are computed as functions of the instantaneous local Reynolds number.

$$
R e_{j}=D_{i} u_{i} \rho / \mu_{i}
$$

where the viscosity $\mu_{j}$ and gas conductivity $k_{j}$ are functions of the local gas temperature $T_{r}$. Standard $f_{i}$ vs $R e_{\text {, correlations }}$ are used for the heater and cooler tubes, and experimental data from Kays and London ${ }^{4}$ were used for the friction in the regenerator matrix. These computations are performed in a separate subroutine, to facilitate substitution of cyclic fluid flow and heat-transfer correlations when available.

Twice per cycle, the gas nlow in the engine reverses, the velocity $u_{i}$ and Reynolds number $R e_{i}$ pass through zero, and the friction factor (in the heater, cooler, and regenerator) goes to infinity. However, this does not cause any computational problems, since the product $f_{i} u_{i}$ in Eq. (9) remains finite,

\section{Finite-Difference Equation for Energy}

In addition to the mass balance and momentum balance presented in Eqs. (8) and (9), we require an energy balance for each node. The rate of change of energy in a typical node $i$ is equal to the net flow rate of (internal and kinetic) energy across its two boundaries, plus the net rate of $P \mathrm{~d} V$ work done on the node by the incoming and outgoing gas streams, plus the heat-transfer rates from the adjacent wall and matrix over the length of the gas node. Therefore,

$$
\begin{aligned}
\frac{\mathrm{d} E_{f}}{\mathrm{~d} t} & =\left[\bar{F}_{i}\left(C_{i} \bar{T}_{i}+1 / 2 \bar{u}_{i}^{2}\right)-\bar{F}_{i+1}\left(C_{t} \bar{T}_{i+1}+1 / 2 \bar{u}_{i+1}^{2}\right)\right] \\
& +\left[\tilde{P}_{i} \bar{V}_{i}-\tilde{P}_{i+1} \bar{V}_{i+1}\right] \\
& +h_{i}\left[S_{w i v}\left(T_{w j}-T_{i}\right)+S_{i m}\left(T_{n+1}-T_{i}\right)\right]
\end{aligned}
$$

The mass balance [Eq. (8)], momentum balance [Eq. (9)], and energy balance [Eq. (1I)] are the three basic gasdynamic equations for each gas node. It would, of coursc, be possible to combine these equations and manipulate them to obtain alternative formulations, e.g., equations for the time derivatives of density $\rho_{j}$, temperature $T_{i}$, and velocity $u_{i}$. But there seems to be no obvious advantage in doing so, since the system of equations will, in any case, have to be solved by numerical techniques. In fact, it is important that the program be able to accommodate abrupt changes in cross-sectional area at the entrance and exit to the heater and cooler. This is done much more readily by keeping the three balance equations in their present form.

\section{Finite-Difference Equations for Metal Temperatures}

In addition to the three gasdynamic equations, we also need heat-balance equations for the wall nodes and matrix nodes. For the wall nodes, the variation of temperature $T_{w y}$ is given by

$$
\begin{aligned}
\frac{\mathrm{d} T_{w i}}{\mathrm{~d} t} & =\left[h_{i} S_{w w}\left(T_{i}-T_{w i}\right)+K_{w, t-1}\left(T_{w i-1}-T_{w i}\right)\right. \\
& \left.+K_{w i}\left(T_{w i+1}-T_{w i}\right)\right] \times C_{w i}^{-1}
\end{aligned}
$$

where $K_{w}$ is the wall's thermal conductance between the center of node $i$ and the center of node $i+1$, and $C_{10}$ is the heat capacitance of wall node $i$.

For the corresponding matrix heat balance, the axial conduction term was omitted. For a typical fine-wire matrix (such as steel wool or stacked screens), with most of the wires oriented normal to the flow direction, it was felt that the axial conduction is negligible compared to the wall conduction and to the convective heat transport. Therefore, the variation of 
matrix temperature is given by

$$
\frac{\mathrm{d} T_{m i}}{\mathrm{~d} t}=\frac{h_{i} S_{m i}\left(T_{i}-T_{m i}\right)}{C_{m i}}
$$

where $C_{m}$ is the heat capacitance of the regencrator matrix in node $i$.

\section{Integration Technique}

The five balance equations $(8,9$, and $11-13)$ just discussed, along with their ancillary algebraic equations (2-7), constitute the basic system of coupled differential equations to be integtated by the program. A number of different numerical integration techniques (e.g., Newton and fourth-order RungeKutta) were tried, but the one which seemed to work most stably was a modified "leapfrog" technique, similar to one used by Physics International in their PISCES code. ${ }^{5}$ In essence, it uses the values of the variables at time $t$, to compute time derivatives. These derivatives are then used to compute the variables values at time $t+\mathrm{d} t$ from their values at time $t-\mathrm{d} t$.

The preceding leapfrog technique was used both with a fixed time step and with a variable time step. As in all explicit finite-difference computations, there is a maximum time step beyond which the program becomes mathematically unstable and the problem blows up (diverges). For our main program, which uses the full momentum balance (including inertial resistancc to flow acceleration), the maximum time step is dictated primarily by the sonic transit time across the length of a node. Stable operation was obtained as long as the time step did not exceed $\sim 30 \%$ of the smallest valuc of "node length divided by local sound velocity" $\left(L_{i} / \sqrt{\gamma_{i} R T_{i}}\right)$. The mathematical accuracy of the method appears to be good, since the results are especially unchanged by order-ofmagnitude reductions in step size.

\section{Node-Boundary Values of Flow Rate and Pressure}

Consider a time $t$ for which we have previously computed the mass $m_{i}$, momentum $M_{i}$, and energy $E_{1}$ for each node. From these, we can calculate the density $\rho_{j}$, velocity $u_{\text {, }}$, temperature $T_{i}$, pressure $P_{c}$, volumetric flow rate $V_{i}$, and mass flow rate $F$, at the center of each gas node.

In addition to these, the three gasdynamic equations (8, 9 , and 11 ) also require the values of these variables at the interface boundaries between adjacent gas nodes. For the volumetric flow rate at the boundary between nodes $i-1$ and $i$, we employ a volume-weighted average of the adjacent nodecenter values:

$$
\tilde{V}_{t}=\frac{A_{j-1} L_{i-1} V_{i}+A_{i} L_{i} V_{t-1}}{A_{i-1} L_{j-1}+A_{i} L_{j}}
$$

More refined interpolation schemes, using parabolic and cubic curve-fits of nearby node-center values, were tried intially. However, the results did not warrant the additional computing effort required.

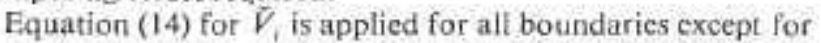
the expansion space/heater interface and the compression space/cooler interface. For these it cannot be used, since the flow rates at the center of the end nodes are essentially undefined.t Instead, the boundary flow rates for those two nodes are compured by extrapolation:

$$
\begin{gathered}
\bar{V}_{2}=2 V_{2}-\hat{V}_{2} \\
\hat{V}_{n}=2 V_{n-1}-\hat{V}_{n \rightarrow 1}
\end{gathered}
$$

IThe momentam balance equation (9) is not applied to the two end nodes.
The pressures at typical nodal boundaries are also computed as volume-weighted averages, analogous to Eq. (14):

$$
\tilde{P}_{i}=\frac{A_{i-1} L_{i-1} P_{i}+A_{i} L_{i} P_{i-1}}{A_{i-1} L_{i-1}+A_{i} L_{i}}
$$

This applies to all internal boundaries within the heater, regenerator, and cooler, where no change of arca occurs. However, at the entrance and exit to the heater and cooler, an additional expansion or contraction term is included, to account for the pressure change due to an abrupt area change.

\section{Stability Problem}

In addition to the preceding expression for $\vec{V}_{7}$ and $\tilde{P}_{1,}$ the gasdynamic equations $(8,9$, and 11$)$ require node-boundary values for gas temperature $T_{i}$ and density $\phi_{\text {, to compute the }}$ mass flow rates, momentum flow rates, and energy flow rates across the nodal boundaries. The simplest and seemingly most logical way of computing the preceding node-boundary variables is by interpolation of the adjacent node-center values of those variables. For example, a volume-weighted averaging scheme similar to Eqs. (14) and (17) could be employed. However, when this was first tried, a condition of mathematical instability was encountered. This took the form of spatial oscillations of various variables. For example, the nodal temperature profile at a given time would exhibit alternating high and low values around a smooth mean curve. This pattern would persist over many time steps, grow in amplitude, and eventually blow up.

The oscillation problem just described is not new but has been observed before in other finite-difference programs (e.g., Ref, 5), Something must be added to such programs to stabilize them and avoid blowups.

\section{Stabilization Schemes}

One such stabilization scheme, used in PISCES and in other programs, is the "donor-cell" concept. In the present case this means that, at each time step, the values of $T_{\text {, }}$ and $\rho_{\text {, at each }}$ boundary are set equal to the variables' node-center values at the adjacent upstream node. For each node boundary, the program tests for the sign of the previously computed volumetric flow rate $\hat{V}$, to determine which is the upstream node. This scheme, also known as "upwind differencing," has been widely used in other Muid-dynamic programs.

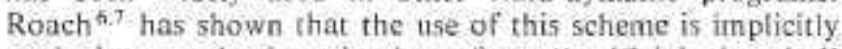
equivalent to the introduction of an "artificial viscosity" term.

Physically, the foregoing scheme appears to be artificial and to make less sense than the previously mentioned averaging schemes, since the actual node-boundary values must generally be intermediate between the adiacent nodecenter values. But mathematically, the donor-cell scheme worked (i.e., gave stable solutions); the simple averaging schemes did not,

Because of concern about the physics of the donor-cell concept, an atternative stabilization scheme was conceived and tried out in our program, Let 15 call this the "yariables-

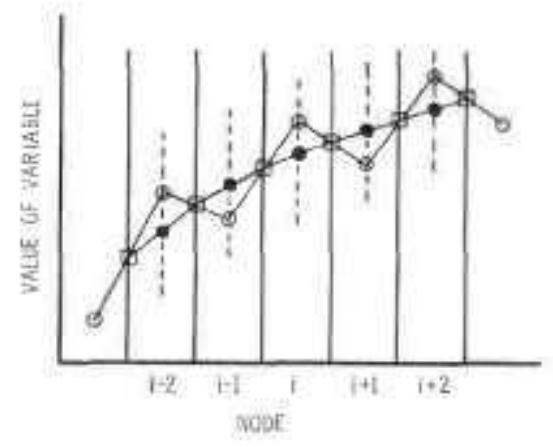

Fig. 2 Variablessmouthing scheme. 
smoothing" scheme. Suppose that a given variable has developed an oscillatory spatial profile, with its nodal values alternating above and below some mean curve. Since the preceding oscillations are merely a numerical artifact of the finite-difference scheme and are not physically real, we wish to smooth out these oscillations, while preserving the shape of the mean curve. Our scheme for doing this is illustrated in Fig. 2 for a number of nodal positions.

The open circles represent the computed node-tenter values, exhibiting typical oscillatory behavior. The open squares represent the node-boundary values, obtained by taking a volume-weighted average of the adjacent node-center values [as in $\mathrm{Eq} .(14)]$. By then taking an arithmetic average of the two boundary values for each node, we obtain corrected node-center values, which are represented by the solid circles in Fig. 2. In practice, when this stabilization scheme is used at every time step, the oscillations never have a chance to build up to any significant amplitude. As a result, the ratios of corrected-to-uncorrected center-node values are all very close to unity.

Both the "donor-cell" scheme and the "variablessmoothing"t scheme were tried in our program, and each was found effective in preventing the buildup of noticeable oscillations or instabifities. The two stabilization schemes appeared to yield identical results. In addition to their individual use, the two schemes can be combined for added stability. The high degree of stability was evidenced when the program was applied to a free-piston problem in which piston collisions temporarily caused violent oscillations in the gas flow rates, but the solution did not diverge.

\section{Alternative Inertialess Program}

When the program just described was applied to typical Stirling engine problems, with the fluid initially at rest in all nodes, it was found that the solutions rapidly home in on a repetitive cycle, with no discernible difference berween corresponding plots for cycle 2 and cycle 3. Although this might lead one to conclude that these early plots represent the steady-state cycle, this is only partially true. It is true that the cyclic plots of flow rates, pressures, and temperatures vary very little from their ultimate, steady-state form and that the mechanical power output per sycle assumes an almost constant value after only a very few cycles. But although the steady-state power output can be determined in only a few cycles, the heat input and the efficiency cannot. This is so because the massive regenerator wall nodes and (to a lesser extent) the matrix nodes have much higher heat capacitances than the corresponding gas nodes and, therefore, take a long time to equilibrate from the initial linear temperature profiles that we had arbitrarily assumed.

To find the complete steady-state solution, with stabilized metal temperature profiles, a much more efficient (even if less accurate) method of solution is required to avoid excessive computation cimes. One way of achieving this is to use an inertialess formulation of the basic equations for rapidly homing in on the steady-state cycle. Once the metal temperatures have stabilized, the more exact inertial equations can then be used for obtaining the final steady-state solution. The inertialess equations can be solved much more rapidly than the inertial equations, because the maximum stable time step is no longer limited by the sonic transit time per node.

In the inertialess formulation, the basic differential cquations for conservation of mass [Eq. (8)] and energy [Eq. (11)) are the same as in the inertial case, but the momentum balance [ $\mathrm{Eq} .(9)]$ is replaced by its steady-state equivatent. In this replacement, the nodal pressure drop at any instant is assumed to equal the nodal friction loss resulting from the instantaneous flow rate. Thus,

$$
\bar{P}_{i}-\bar{P}_{j+1}=\left(f_{i} L_{i} / D_{i}\right)\left(1 / 2 \rho_{i}\left|u_{i}\right| u_{t}\right)
$$

with appropriate corrections for abrupt expansions or contractions, where necessary. Replacement of Eq. (9) by Eq.
(18) is tantamount to assuming that the inertial forces are negligible compared to the friction forces. This assumption is likely to be more valid at low frequencies than at high.

With the foregoing change, the previous method of solution can no longer be employed, since Eq. (9) for the rate of change of momentum (or flow rate) has been eliminated. The remaining equations could be solved in an implicit manner, but this can be quite time-consuming, particularly if matrixinversion or successive-approximation solutions are employed.

Fortunately, by taking advantage of the fact that spatial pressure variations are small compared to the temporal variations, it was possible to devise an algorithm for solving the inertialess set of differential equations, along with their ancillary algebraic relationships, in an expeditious manner. The algorithm uses an explicit, noniterative method of solution for each time step. Since the time step size in this formulation is governed by the energy equation rather than the mornentum equation, considerably larger time steps can be used. For the problem just described, the inertialess program decreased the computer time per machine cycle by a factor of 20.

This increase in computing speed is useful not only for finding the steady-state solution with stabilized metal temperatures but also for preliminary design studies, where we wish to compare a large number of design alternatives and are willing to sacrifice absolute accuracy for speed. For our illustrative problem (i.e., at $22 \mathrm{~Hz}$ ), the solutions from the inertialess program exhibit very good agreement with those of the full inertial program.

\section{Accelerated Convergence}

Even the higher speed of the inertialess program, however, is not by itself adequate for equilibrating the wall and matrix temperatures in a reasonable number of cycles. Although the engine's power output can be determined quite quickly, determinations of its efficiency would require prohibitive computing time, unless an effective accelerating seheme is used.

The accelerating scheme evolved for the SNAP program is to artifically divide the heat capacity of the regenerator matrix and wall by acceleration factors which diminish with time. For example, we employed wall and matrix acceleration factors starting at values of 2500 and 250 , respectively, at the beginning of the run, and diminishing parabolically to unity at the end of the 25 th cycle.

The preceding acceleration scherne leads to quite rapid convergence to the steady-state cycle. Even faster convergence could, of course, by achieved if the initial regenerator wail temperature profile, instead of being a tinear ramp between heater and coolet, were based on previous experience. The program has the option of automatic restart from the end of a previous problem.

For problems with leakage through seals, an additional accelerating scheme is needed. Without such a scheme, many cycles of computation would be requited for the gas in the large bounce space to equilibrate at its steady-state temperature. In the scheme used in SNAP, the net energy changes of the gas in the bounce space and in the gas spring are determined after each cycle. From these, the program computes what temperature changes in those two nodes would have been required to mull the observed energy changes during the previous cycle. These two temperature changes are then introduced as step changes before starting the new eycle. Of course, this scheme does not work instanteously, because (as a result of leakage) the imposed temperature changes, in turn, affect the compression space and the rest of the working fluid. But if the scheme is applied at the end of each cycle, the gas spring and bounce space temperatures equilibrate rather quickly. The two acceleration schemes, i.e., the earlier one for cquilibrating the wall temperatures and the foregoing one for equilibrating the gas springs, can be applied simultancously. 
Table I Leakage paths through seals

\begin{tabular}{lccc}
\hline \hline Seal between & Diameter, ct & Length, cm & Gap width, mm \\
\hline Expansion and compression space & 5.71 & 0.965 & 0.0244 \\
Compression and bounce space & 5.71 & 4.876 & 0.0231 \\
Compression and bounce space ${ }^{\mathrm{a}}$ & 2.21 & 3.49 & 0.0203 \\
Cias spring and bounce space" & 2.21 & 2.04 & 0.0203 \\
\hline \hline
\end{tabular}

${ }^{3}$ At the esarer of the piston rod seal, thete is a circumferential groove, which is connected to the bounce space (not shown in Fig. 1).

\section{Illustrative Problem}

An illustrative prolem was analyzed using the methodology described previously. This consisted of an inertialess analysis ( 25 accelerated cycles plus 2 unaccelerated cycles) followed by an inertial analysis for an additional two cycles. The final results of the two analyses were in good agreement. The results for the last cycle of the inertial analysis are depicted in Figs. 4-14.

A schematic view of the machine topology and of the nodal arrangement was presented eartier in Fig. 1, Although that figure shows only a single string of gas nodes, in the actual machine there are 22 parallel heater tubes and 135 parallel cooler ducts. These are connected to a single annular regenerator, filled with a wire matrix of $0.05 \mathrm{~mm}$ diameter and having a matrix porosity of $91 \%$.

The power piston has a stroke of $2.54 \mathrm{~cm}$, and the displacer stroke is $2.66 \mathrm{~cm}$. The two pistons move with a frequency of $21.9 \mathrm{~Hz}$, and the displacer leads the piston by a phase angle of $70.4 \mathrm{deg}$. With both pistons at their midstroke position, there is a gap of $1.775 \mathrm{~cm}$ bet ween the two pistons and a gap of 3.19 $\mathrm{cm}$ between the displacer and the cylinder head. In addition to the volumes which can be swept by the pistons, there is a residual volume of $4.0 \mathrm{~cm}^{3}$ in the expansion space when the displacer meets the cylinder head and a residual volume of $23.5 \mathrm{~cm}^{3}$ in the compression space when the two pistons meet.

The prescribed piston motions are illustrated in Fig. 3. The lower curve represents the power piston, the middle curve the displacer piston, and the upper bold line the cylinder head. The expansion space lies between the displacer and the cylinder head, and the compression space between the power piston and the displacer. As can be seen, during one part of the eycle the power piston and displacer almost touch, and the compression space almost vanishes (except for the residual volume),

Finally, the problem considers both continuous-flow leaks, through piston and piston rod seals, and periodic leaks through centering ports. $\$$ The continuous leakage takes place through four annular seals, whose dimensions are shown in Table 1.

The periodic leakage takes place through two sets of centering ports. The ports connecting the compression space with the bounce space are aligned when the power piston is $0.343 \pm 0.051 \mathrm{~cm}$ below its midstroke position; the ports connecting the gas spring with the bounce space are aligned when the compression space length is $1.480 \pm 0.132 \mathrm{~cm}$. Each set of ports is aligned twice during each cycle. When the ports are aligned, they have a conductance equal to that of an oritice of $0.107 \mathrm{~cm}$ diameter.

The preseribed wall temperatures are $593^{\circ} \mathrm{C}$ for the heater and expansion space and $31^{\circ} \mathrm{C}$ for the cooler and compression space. Initially, the regenerator wall and matrix were assumed to have a linear temperature gradient between their hot and cold ends. In general, the program treats the temperatures of the hot walls and cold walls as fixed and allows the temperatures of the regenerator's wall nodes and matrix nodes to float. Occasionally, the program was run with floating heater wall temperatures, with prescribed heat input rates.

\footnotetext{
Such centering ports are required in free piston engines, to prevent the displacer and piston motion from drifting off their desired midstroke positions.
}

The problem was started with a uniform pressure of 60 bars, with the fluid initially at rest in all nodes, and with an initial cycle angle of $72 \mathrm{deg}$. This was an arbitrary choice. The fluid flow solutions rapidly home in on a steady-state cycle, regardless of starting angle and initial flow rate.

\section{Cyclic Pressure Profiles}

The piston motions shown earlier in Fig. 3 produce the cyclic pressure variation depicted in Fig. 4. Actually, that figure presents seven different curves, representing pressures at different locations ranging from the expansion space to the compression space. However, these curves are so close together that they are indistinguishable on the scale of the figure. In other words, at the prescribed operating frequency of $22 \mathrm{~Hz}$, the spatial variation of pressure is very small compared to its temporal variation.

The two vertical lines in Figs. 3-7 depict the points of maximum and minimum pressure. As shown in Fig. 3, these are close to the points of minimum and maximum gas volume. However, they do not quite coincide, because of changes in the mean gas temperature. As can be seen, the pressure wave lags the power piston motion by $30 \mathrm{deg}$.

Figure 5 shows the cyclic variation of the pressure difference between various gas nodes and the center of the regenerator. These pressure differences include the friction losses, the expansion and contraction effects at the ends of the heater and cooler, and the acceleration and deceleration forces required to oyercome the fluid's inertia and cause the changes in fluid flow rates shown below.

\section{Cyclic Mass-Flow-Rate Profiles}

When a problem is first started from arbitrary initial conditions, the solution exhibits rather violent startup oscillations, but these die out suprisingly fast. By the second half of eycle 1 , the eventual flow pattern is quite well established. It is noteworthy that the initial startup oscillations have a period approximately equal to the roundIrip transit time of a sound wave through the total gas passage. Sometimes these sonic oscillations, in much milder form, also make a brief appearance during the steady-state cycle after reversals of the flow direction.

The cyclic flow yariation, at nine points in the engine, is depicted in Fig. 6. The period of positive flow (hot end to cold end) exceeds the period of negative flow at all points, and the

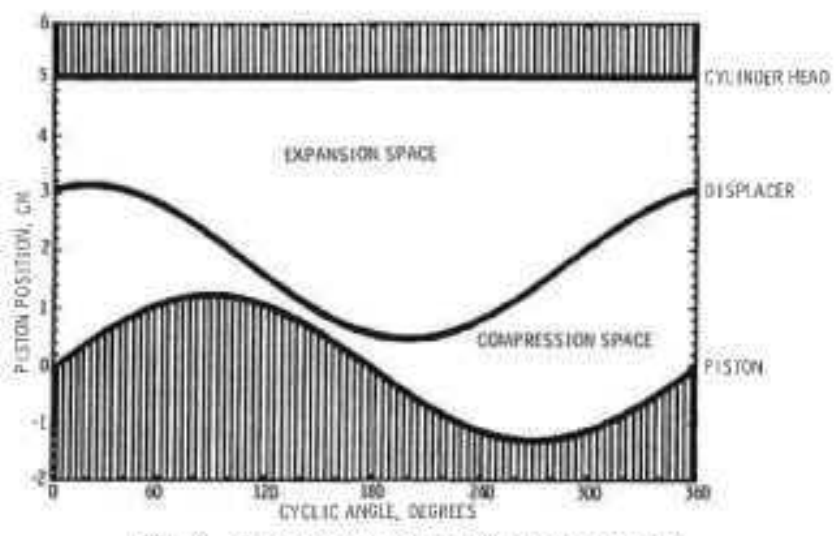

Fig. 3 Cyclic motions of piston and displacer. 


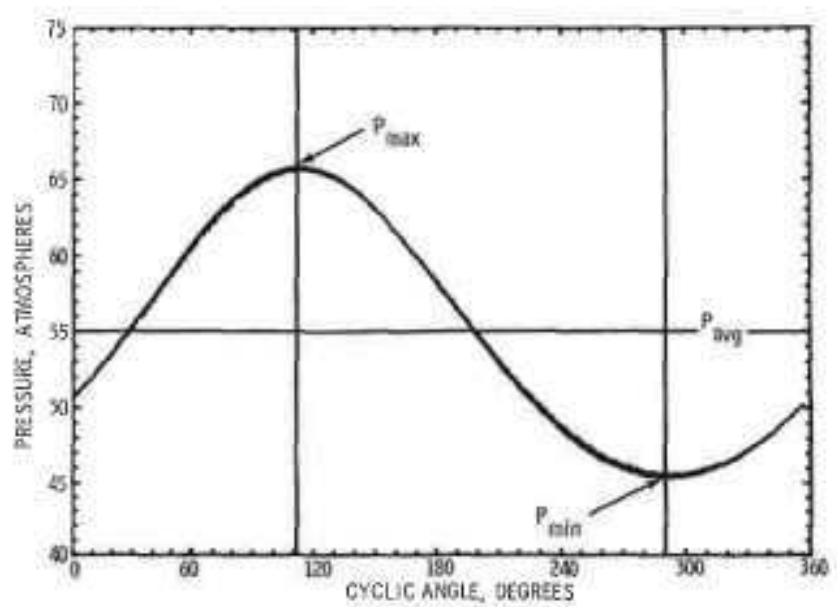

Fig. 4 Cyclic pressure variation.

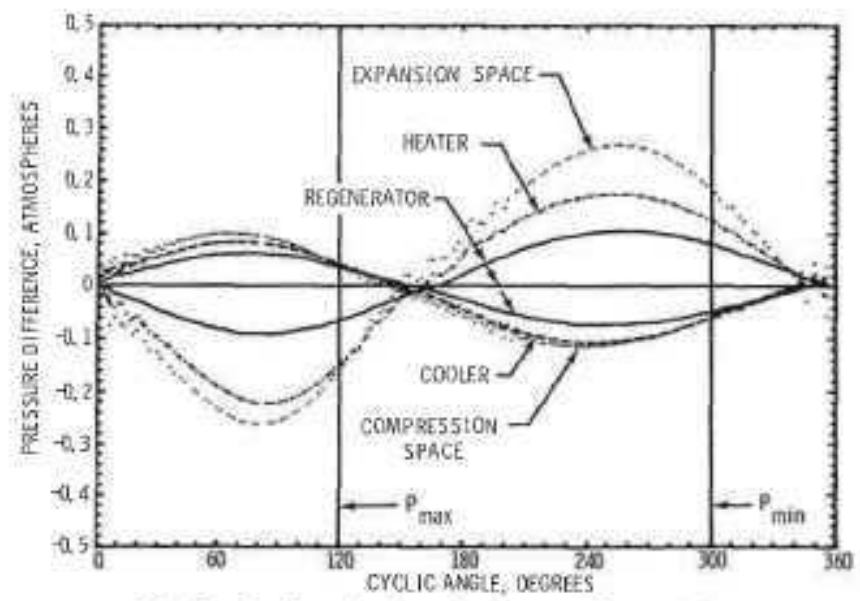

Fig. 5 Cyclic variation of pressure differentials.

mass flow rate is far from uniform most of the time. In fact, there are times when positive and negative flows coexisf. For example, at a cyclic angle of $155 \mathrm{deg}$, the gas volume is expanding, and the pressures and densities are decreasing rapidly. As a result, gas is streaming out of both ends of the regenerator. The reverse situation exists at a cyclic angle of 350 deg.

As can be seen, flow reversal (in either direction)starts at the compression-space end and proceeds toward the expansion space. There are two points in the cycle when all mass now rates are approximately equal. These occur near the points of maximum and minimum pressure, when the nodal gas densities are not changing.

\section{Cyclic Temperature Profiles}

Figure 7 shows the cyclic variation of gas temperatures in the expansion space, in two heater nodes $(3,6)$, five regenerator nodes $(9,11,13,15,17)$, two cooler nodes $(20$, 23), and the compression space, as well as the variation of matrix temperatures at the same five regenerator locations. Heater and cooler wall temperatures are also shown in the figure.

Since heat-transfer rates to the expansion- and compression-space walls are relatively small, these spaces are close to adiabatic. Therefore, their temperature variations are caused primarily by compression and expansion effects. Both temperatures reach a maximum near the time of maximum pressure and a minimum near the time of minimum pressure.

The variations of gas temperatures in the heater and cooler nodes displayed in Fig. 7 show that during part of the cycle the gas in the heater is actually hotter than the heater wall, and during part of the cycle the gas in the cooler is colder than

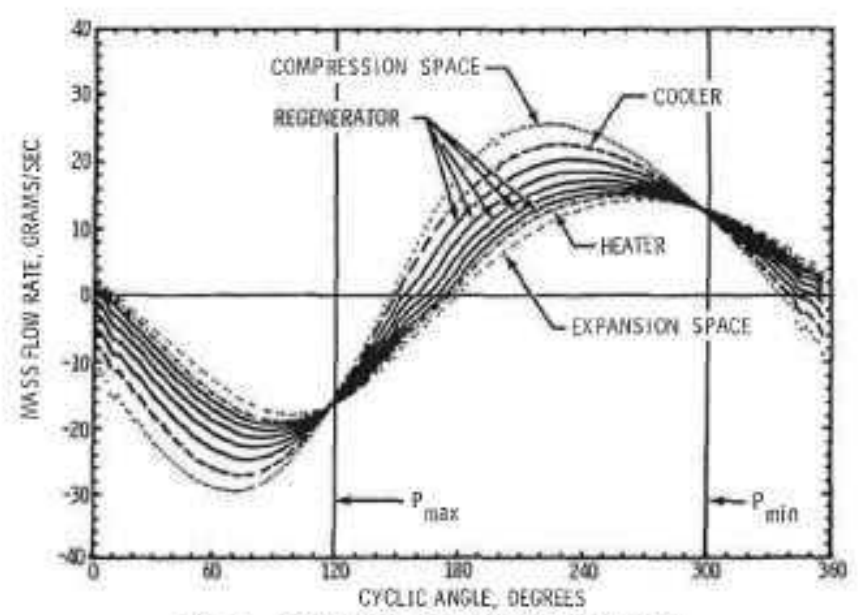

Fig. 6 Cyelic variation of mass flow rates.

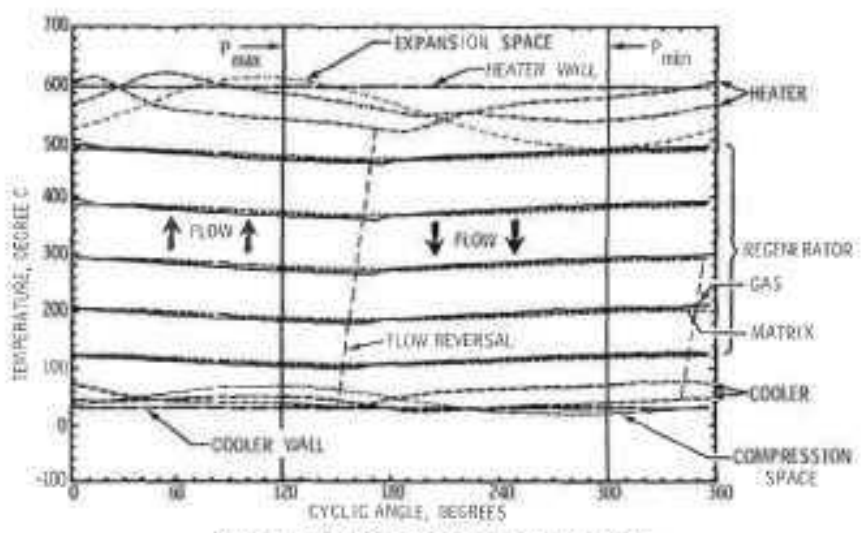

Fig. 7 Cyclic temperature variation.

the cooler wall. This is a source of inefficiency, which results from the fact that the thermal coupling between gas and wall in the expansion and compression spaces is relatively weak.

Finally, Fig. 7 displays five solid eurves, showing the variation of gas temperature in the regenerator (at nodes 9 , $11,13,15,17)$, accompanied by five short-dash curves, thowing the variation of matrix temperature at the stame five locations. The time of flow reversal at various points of the regenerator is indicated by the two slanting dashed lines. The cyclic variation of the gas temperature and of the matrix temperature is essentially the same at all regenerator locations. During somewhat more than half of the cycle, the gas is hotter than the matrix, and heat is being stored in the matrix. During the remainder of the cycle, the warmer matrix returns the stored heat to the cooler gas. The relative temperature reyersals do not occur simultaneously at all locations. In both directions, the temperature reversal starts at the cold end and propagates to the hot end.

For the given regenerator design parameters, the matrix temperatures are far from constant. As shown, they undergo quite noticeable periodic swings as the result of the cyclic heat addition and removal. The temperature fluctuation of the regenerator wall is much smaller, because of the latter's much lower surface-to-volume ratio. These temperatures have not been plotted, since their cyclic variation would not be discernible on the scale of the figure.

\section{Spatial Temperature Profiles}

In addition to the gas temperatures ${ }^{t}$ eyclic variation, it is also of interest to examine their spatial variation at various times in the cycle. Such spatial temperature profiles are displayed in Fig. 8 as a function of nodal position. Thirty-six curves are shown, at intervals of 10 deg of cyclic angle. To promote clarity, each curve is offset by $15^{\circ} \mathrm{C}$ from its 


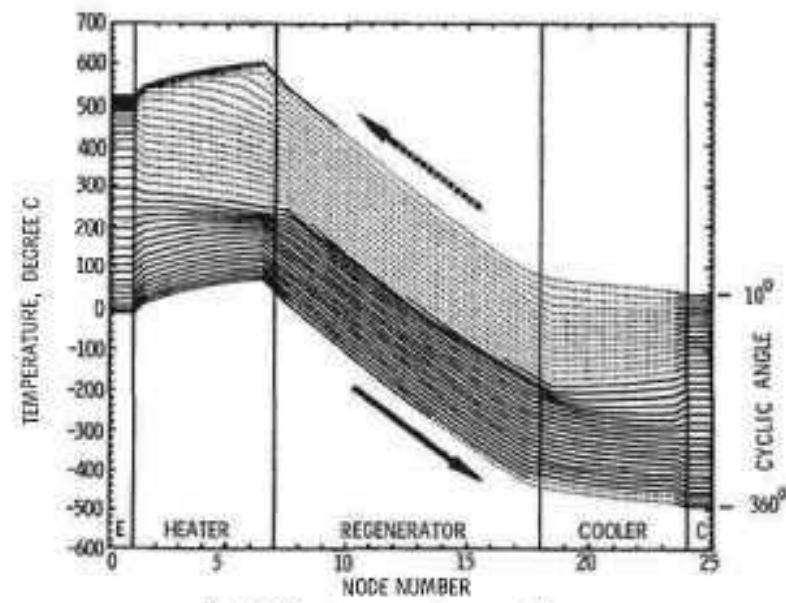

Fig. 8 Gas temperature profiles.

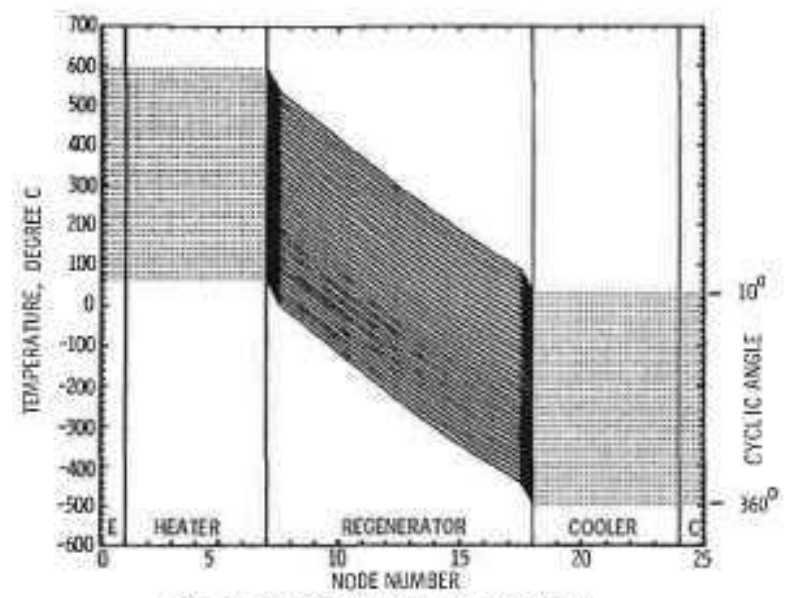

Fig. 9 Matrix temperature profiles.

predecessor. Thus, the temperature scale shown is an absolute scale for only the first (i.e., topmost) curve; it is only a relative scale for the other curves.

To help interpret the curve, flow from left to right is denoted by solid curves, and flow from right to left is denoted by dotted curves. Close-spaced curves indicate rising temperatures. Wide spacings denote diminishing temperatures. As can be seen, the spatial gas temperature profiles vary considerably during the cycle. This is particularly true in the heater and cooler. After flow is well established, the gas temperature rises in the flow direction in the heater and falls in the flow direction in the cooler. This is not true immediately after flow reversal, and some time elapses before the proper temperature profiles are established.

The spatial temperature profiles of the regenerator matrix are presented in an analogous manner in Fig. 9. For each curve, the dotted extension represents the corresponding hotand cold-wall temperatures. As can be seen, the spatial matrix-temperature profiles show much less variation during the cycle than the gas-temperature profiles. This is because the matrix nodes have a much higher heat capacitance than the gas nodes. This is even more true of the analogous wall temperature profiles. Those profiles (not shown) look very similar to the matrix profiles, with no discernible cyclic variation.

It will be noted that the matrix temperature profiles shown in Fig. 9 differ only a little from the initially assumed linear ramps between the hot- and cold-wall temperatures. Yet, determination of this small difference, essentially a slight curvature and a small tilt, requires a great deal of computation and is essential for obtaining the steady-state solution.

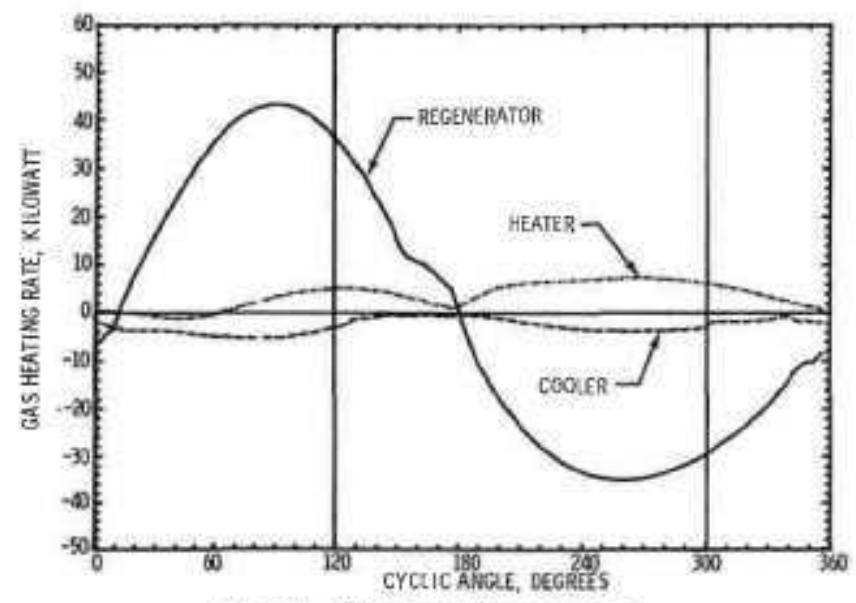

Fig. 10 Cyclic beat-flow variation.

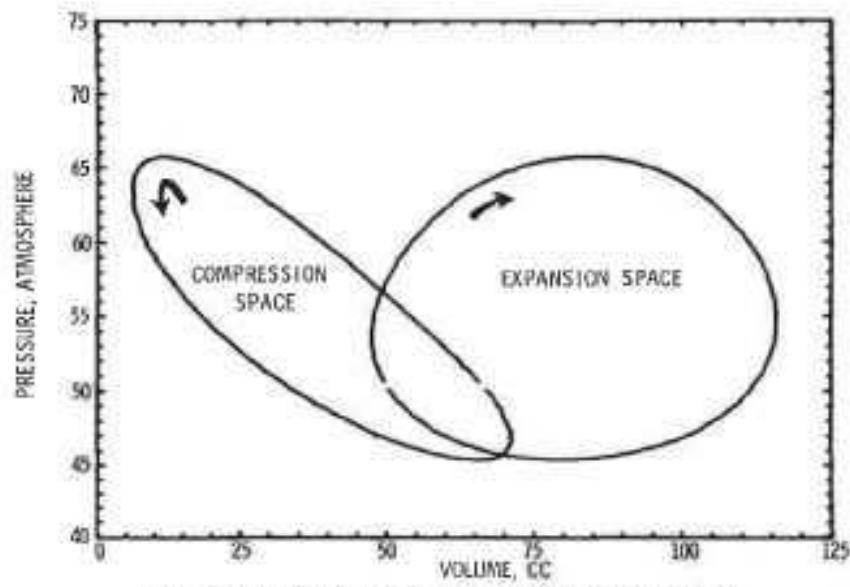

Fig. 11 Pressure-volume diagrams for end nodes.

\section{Cyclic Heat-Flow Profiles}

Figure 10 shows the cyclic variations of the heat flow rates from various components to the working fluid. The dot-dash curve represents heat flow from the heater and expansionspace wall to the gas; the dashed curve represents heat flow from the cooler and compression-space wall to the gas; and the solid curve represents heat flow from the regenerator (matrix plus wall) to the gas.

As can be seen, the heat flow to and from the regenerator is much greater than the heat flows from the heater and to the cooler. This is true of any well-designed Stirling engine. Also, we note that all three curves exhibit irregularities at the points of flow reversal, where the local flow velocities (and therefore the heat-transfer coefficients) pass through zero. Finally, we see that the matrix-to-gas heat flow is briefer and has a higher peak than the gas-to-matrix beat flow.

\section{Mechanical Power Output}

Figure 11 presents the pressure-volume diagrams for the expansion space and the compression space, and Fig. 12 shows the piston force vs position diagrams for the displacer and the power piston. As shown by the arrowheads in Fig. 11, time variation is clockwise along the expansion-space curve and counterciockwise along the compression-space curve. During a cycle, the working fluid does net work in the expansion space and has work done on it in the compression space, Since the area enclosed by the expansion-space curve is larger than that enclosed by the compression-space curve, the heat engine has a positive power output.

In Fig. 12, the force exerted by the working fluid on each piston is plotted vs the piston's position. The area enclosed by each curve represents the work which the gas does on the 


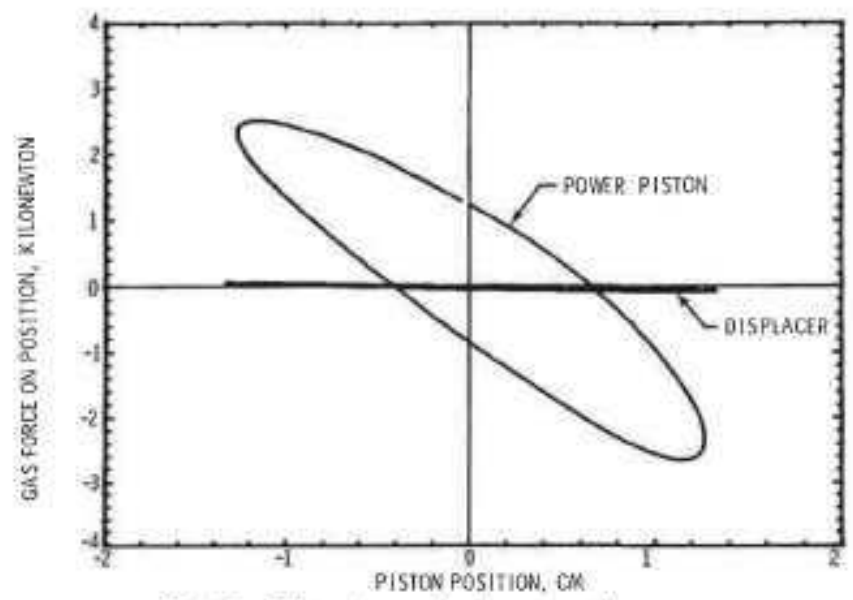

Fig. 12 Piston force-displacement diagrams.

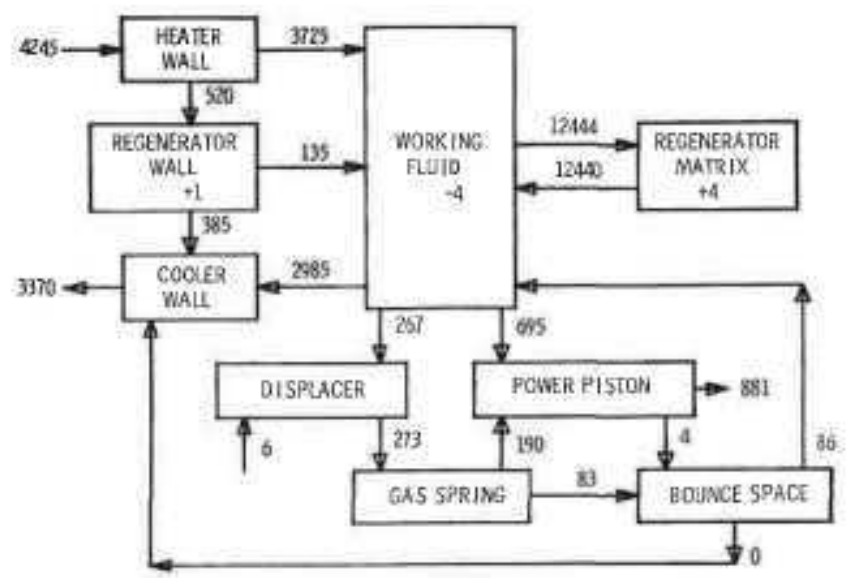

EFFICIENCY $+884425=20,7 \mathrm{~K}$

Fig. 13 Energy balance for lasi cycle.

piston during the cycle. The area enclosed by the displacer curve is close to zero, as it should be. Virtually all of the mechanical power goes to the power piston for transmission to the load. The fact that the net force on the displacer is always close to zero shows that the disciplined-piston motions analyzed in this problem are consistent with free-piston operation, since in a free-piston machine the net displacer work must be zero.

\section{Energy Balance}

The overall energy balance for the preceding problem is summarized in Fig. 13. All cyclie energy changes are expressed as rates of change (in watts) averaged over the eycle. As can be seen, during the cycle the working gas receives heat at an average rate of $3725 \mathrm{~W}$ from the heater and expansionspace walls and $135 \mathrm{~W}$ from the regenerator wall; it loses heat at a rate of $2985 \mathrm{~W}$ to the cooler and compression-space walls. In the same period, the gas sees an average heat flow rate of $12,444 \mathrm{~W}$ to the regenerator matrix and $12,440 \mathrm{~W}$ from the matrix, giving a net heat-transfer rate of $4 \mathrm{~W}$ from the gas to the matrix.

In addition, there is net heat transfer from the bounce space to the compressor space at a rate of $86 \mathrm{~W}$, averaged over the cycle. This is due to net heat transport as a result of cyclic leakage between those spaces. Although the forward and backward mass flows must (and do) balance over the steady. state cycle, the accompanying energy flows need not balance, since the compression space and bounce space can both transfer energy to or from other parts of the system.

Summing the foregoing energy transfer rates, we obtain a net energy input rate to the working fluid of $958 \mathrm{~W}$, averaged over the cycle. In the same period, the working fluid does mechanical work at the rate of $267 \mathrm{~W}$ on the displacer and 695 W on the power piston, for a combined mechanical output rate of $962 \mathrm{~W}$.

The difference of $4 \mathrm{~W}$ (or $0.4 \%$ ) between the preceding sums does not represent a discrepancy in the working fluid energy balance. That energy balance closes much better than $0.4 \%$. The $4-\mathrm{W}$ difference is an indication of how close the solution is to the steady-state cycle. Other indicators shown in Fig. 13 are the rates of enthalpy change of the regenerator wall $(1 \mathrm{~W}$, or $0.5 \%)$ and of the regenerator matrix $(4 \mathrm{~W}$, or $0.003 \%$ ). The smallness of these differences demonstrates the effectiveness of the acceleration schemes in homing in on a steady-state solution from arbitrary starting conditions.

In this connection, it should be pointed out that the $135-\mathrm{W}$ heat flow rate from the regenerator wall to the working fluid is consistent with steady-state operation. It is balanced by the difference between the 520 -W heat flow from heater wall to regenerator wall and the 385 .W heat flow from regenerator wall to cooler wall.

As mentioned earlier, the working fluid does mechanical work on the displacer at an average rate of $267 \mathrm{~W}$. This rather large quantity is not a loss to the system, since much of it is transferred to the power piston via the gas spring (i.e., the cavity within the power piston shown in Fig. 1). Power is transferred because of the phase difference between the two pistons.

It was found that, in problems with all leakages set to zero, all of the energy which the gas spring receives from the displacer is returned to the power piston. But this is not true for problems with nonzero leakage. As shown in Fig. 13, of the $273 \mathrm{~W}$ received from the displacer, only $190 \mathrm{~W}$ are transmitted to the power piston. The other $83 \mathrm{~W}$ are transferred from the gas spring to the bounce space, as the result of leakage between the two spaces. Again, mass transfer due to leakage is zero over the cycle, but energy transfer is not.

The $83 \mathrm{~W}$ lost to the bounce space is returned to the compression space via other leakage paths. Although the energy is therefore not lost to the system, it causes a very serious decrease in power output and efficiency, since mechanical energy is converted into low-temperaturc heat. It was found that, other things being equal, the engine described in this paper would have a power output of $1238 \mathrm{~W}$ without leakage and only $881 \mathrm{~W}$ with the postulated leakage paths, with a corresponding reduction of system efficiency from $28.7 \%$ to $20.7 \%$. This demonstrates the importance of reducing the seals ${ }^{*}$ gap widths to the lowest possible values, since leakage is proportional to the cube of the gap width.

\section{Motion of Gas Particles}

As mentioned earlier, once a given problem has been analyzed by the Eulerian SNAP program, the results obtained can readily be used to generate a family of Lagrangian plots. One such plot for the preceding problem is shown in Fig. 14. Here the nodal positions of specific gas particles are plotted as a function of cyclic angle. The heater, regenerator, and cooler are shown as fixed nodes ( 1 through 24). The expansion and compression spaces are depicted as variable nodes (of arbitrary scale), to represent their volume variation as the result of piston and displacer motions during the eycle. These volume variations cause the gas particle motions represented by the curves.

The figure presents the nodal paths of 19 gas particles, at intervals of $5 \%$ of the total gas mass in the working space. Thus, the band between any two neighboring curves reprsents $5 \%$ of the working fluid. As can be seen, about $20 \%$ of the working fluid never leaves the compression space, and approximately $10 \%$ never leaves the expansion space. No single gas particle moves all the way from the expansion space to the compression space. In fact, for the problem at hand, no single gas particle traverses the entire regenerator during the cycle. One of the gas particle paths shown never leaves the regenerator. 


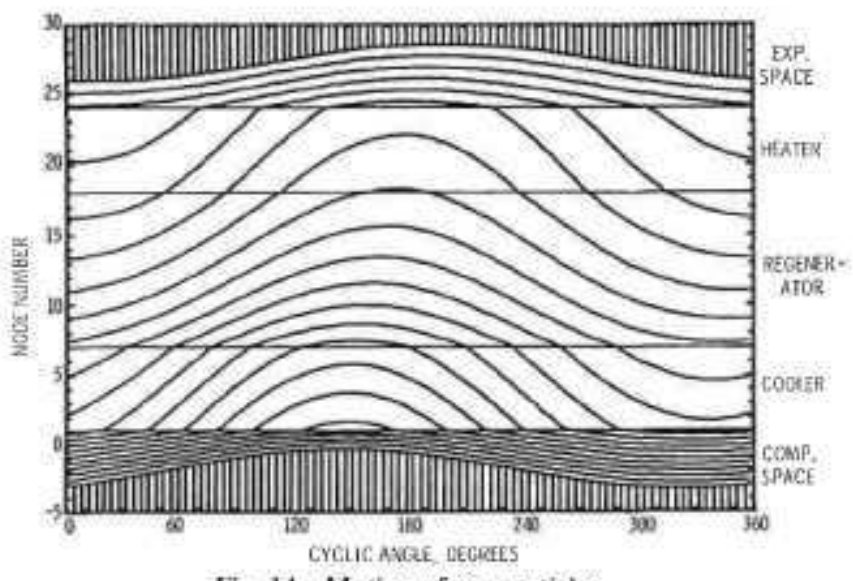

Fig. 14 Motion of gas particles.

\section{Conclusion}

The program just described and illustrated provides a versatile and, it is hoped, useful tool for the analysis of cyclic thermodynamic machines. It should be readily adaptable to other machine topologies and to future refinements of the analytical modet. The program listing is available by contacting the author.

\section{Acknowledgments}

The author takes pleasure in expressing his appreciation for the assistance of M.B. Eck, M. Mukunda, H. Sookiazian, and E. Vogel, all of Fairchild Industries, in carrying out the work just described.

\section{References}

'Goldwater, B. and Mortow, R.B., "Demonstration of a FreePiston Stitling Linear Alternator Power Conversion System," Proceedings of the 12 th $I E C E C$, Vol, 2, 1977,

${ }^{2}$ Finkelstein, T., "Thermophysics of Regenerative Energy Conversion," AIAA Paper 67:216, A1AA 5th Aerospace Science Meeting, 1967.

${ }^{3}$ Urieli, 1., "A Computer Simulation of Stirling Cycle Machines," Ph.D. Thesis, Univ, of the Witwatersrand, South Africa, 1977.

"Tong, L.S and London, A.L., "Heat-Transier and Flow-Friction Characteristics of Woven-Screen and Crossed-Rod Matrixes," Transactions of ASME, Vot. 79, Oct. 1957.

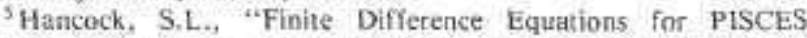
2DELK, A Coupled Euler Lagrange Continuum Mechanics Computer Program," Physics Intemational Co., Tech. Memo, TCAM 76-2, 1976.

${ }^{6}$ Roache, P.J., Computational Fluid Dynamics, Hermosa Publishers, Albuquerque, N. Mex., 1976.

"Roache, P.J., "On Artificial Viscosity," foumal of Computational Physics, Vol, 10, Oct. 1972. 